\title{
A new approach for CMS RPC current monitoring using Machine Learning techniques
}

To cite this article: A. Samalan et al 2020 JINST 15 C10009

View the article online for updates and enhancements.

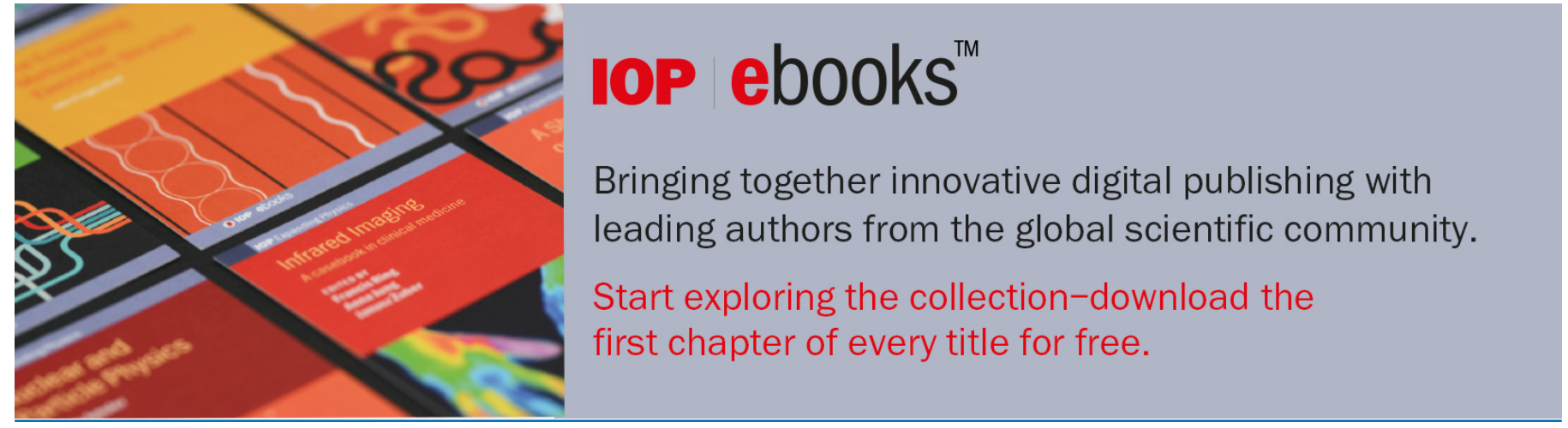


XV Workshop on Resistive Plate Chambers and Related Detectors

10-14 FEBRUARY, 2020

University of Rome Tor Vergata, Rome, Italy

\section{A new approach for CMS RPC current monitoring using Machine Learning techniques}

A. Samalan, ${ }^{a}$ M. Tytgat, ${ }^{a}$ N. Zaganidis, ${ }^{a}$ G.A. Alves, ${ }^{b}$ F. Marujo, ${ }^{b}$

F. Torres Da Silva De Araujo, ${ }^{c}$ E.M. Da Costa, ${ }^{c}$ D. De Jesus Damiao,${ }^{c}$ H. Nogima, ${ }^{c}$

A. Santoro, ${ }^{c}$ S. Fonseca De Souza, ${ }^{c}$ A. Aleksandrov, ${ }^{d}$ R. Hadjiiska, ${ }^{d}$ P. laydjiev, ${ }^{d}$

M. Rodozov, ${ }^{d}$ M. Shopova, ${ }^{d}$ G. Sultanov, ${ }^{d}$ M. Bonchev,${ }^{e}$ A. Dimitrov, ${ }^{e}$ L. Litov, ${ }^{e}$ B. Pavlov, ${ }^{e}$

P. Petkov, ${ }^{e}{ }^{, 1}$ A. Petrov, ${ }^{e}$ S.J. Qian,,${ }^{f}$ C. Bernal, ${ }^{g}$ A. Cabrera, ${ }^{g}$ J. Fraga,,${ }^{g}$ A. Sarkar,${ }^{g}$

S. Elsayed, ${ }^{h}$ Y. Assran, ${ }^{b b, f f}$ M. El Sawy, ${ }^{b b, c c}$ M.A. Mahmoud, ${ }^{i}$ Y. Mohammed, ${ }^{i}$ C. Combaret,${ }^{j}$

M. Gouzevitch, ${ }^{j}$ G. Grenier, ${ }^{j}$ I. Laktineh, ${ }^{j}$ L. Mirabito, ${ }^{j}$ K. Shchablo, ${ }^{j}$ I. Bagaturia, ${ }^{k}$

D. Lomidze,${ }^{k}$ I. Lomidze,${ }^{k}$ V. Bhatnagar,${ }^{l}$ R. Gupta, ${ }^{l}$ P. Kumari, ${ }^{l}$ J. Singh, ${ }^{l}$ V. Amoozegar,${ }^{m}$

B. Boghrati, ${ }^{m, d d}$ M. Ebraimi, ${ }^{m}$ R. Ghasemi, ${ }^{m}$ M. Mohammadi Najafabadi, ${ }^{m}$ E. Zareian, ${ }^{m}$

M. Abbrescia, ${ }^{n}$ R. Aly, ${ }^{n}$ W. Elmetenawee, ${ }^{n}$ N. Filippis,${ }^{n}$ A. Gelmi,${ }^{n}$ G. laselli, ${ }^{n}$ S. Leszki ${ }^{n}$

F. Loddo, ${ }^{n}$ I. Margjeka, ${ }^{n}$ G. Pugliese, ${ }^{n}$ D. Ramos, ${ }^{n}$ L. Benussi, ${ }^{o}$ S. Bianco, ${ }^{o}$ D. Piccolo, ${ }^{o}$

S. Buontempo, ${ }^{p}$ A. Di Crescenzo, ${ }^{p}$ F. Fienga, ${ }^{p}$ G. De Lellis, ${ }^{p}$ L. Lista, ${ }^{p}$ S. Meola, ${ }^{p}$

P. Paolucci, ${ }^{p}$ A. Braghieri, ${ }^{q}$ P. Salvini, ${ }^{q}$ P. Montagna, ${ }^{e e}$ C. Riccardi, ${ }^{e e}$ P. Vitulo, ${ }^{e e}$

B. Francois, ${ }^{r}$ T.J. Kim,${ }^{r}$ J. Park, ${ }^{r}$ S.Y. Choi, ${ }^{s}$ B. Hong, ${ }^{s}$ K.S. Lee, ${ }^{s}$ J. Goh, ${ }^{t}$ H. Lee, ${ }^{u}$

J. Eysermans, ${ }^{v}$ C. Uribe Estrada, ${ }^{v}$ I. Pedraza, ${ }^{v}$ H. Castilla-Valdez, ${ }^{w}$ A. Sanchez-Hernandez, ${ }^{w}$

C.A. Mondragon Herrera, ${ }^{w}$ D.A. Perez Navarro, ${ }^{w}$ G.A. Ayala Sanchez, ${ }^{w}$ S. Carrillo, ${ }^{x}$

E. Vazquez, ${ }^{x}$ A. Radi, ${ }^{y}$ A. Ahmad, ${ }^{z}$ I. Asghar, ${ }^{z}$ H. Hoorani, ${ }^{z}$ S. Muhammad, ${ }^{z}$ M.A. Shah ${ }^{z}$

and I. Crotty ${ }^{a a}$ on behalf of the CMS collaboration

${ }^{a}$ Ghent University, Department of Physics and Astronomy, Proeftuinstraat 86, B-9000 Ghent, Belgium

${ }^{b}$ Centro Brasileiro Pesquisas Fisicas,

R. Dr. Xavier Sigaud, 150 - Urca, Rio de Janeiro - RJ, 22290-180, Brazil

${ }^{c}$ Departamento de Fisica Nuclear e Altas Energias, Instituto de Fisica,

Universidade do Estado do Rio de Janeiro,

Rua Sao Francisco Xavier, 524, BR - Rio de Janeiro 20559-900, RJ, Brazil

${ }^{d}$ Bulgarian Academy of Sciences, Institute for Nuclear Research and Nuclear Energy,

Tzarigradsko shaussee Boulevard 72, BG-1784 Sofia, Bulgaria

${ }^{e}$ Faculty of Physics, University of Sofia,5 James Bourchier Boulevard, BG-1164 Sofia, Bulgaria

${ }^{f}$ School of Physics, Peking University, Beijing 100871, China

${ }^{1}$ Corresponding author. 
${ }^{g}$ Universidad de Los Andes, Apartado Aereo 4976, Carrera 1E, no. 18 A 10, CO-Bogota, Colombia

${ }^{h}$ Egyptian Network for High Energy Physics, Academy of Scientific Research and Technology, 101 Kasr El-Einy St., Cairo Egypt

${ }^{i}$ Center for High Energy Physics, Faculty of Science, Fayoum University, 63514 El-Fayoum, Egypt

${ }^{j}$ Universite de Lyon, Universite Claude Bernard Lyon 1,

CNRS-IN2P3, Institut de Physique Nucleaire de Lyon, Villeurbanne, France

${ }^{k}$ Georgian Technical University, 77 Kostava Str., Tbilisi 0175, Georgia

${ }^{l}$ Department of Physics, Panjab University, Chandigarh 160 014, India

${ }^{m}$ School of Particles and Accelerators, Institute for Research in Fundamental Sciences (IPM),

P.O. Box 19395-5531, Tehran, Iran

${ }^{n}$ INFN, Sezione di Bari, Via Orabona 4, IT-70126 Bari, Italy

o INFN, Laboratori Nazionali di Frascati (LNF), Via Enrico Fermi 40, IT-00044 Frascati, Italy

p INFN, Sezione di Napoli, Complesso Univ. Monte S. Angelo, Via Cintia, IT-80126 Napoli, Italy

${ }^{q}$ INFN, Sezione di Pavia, Via Bassi 6, IT-Pavia, Italy

${ }^{r}$ Hanyang University, 222 Wangsimni-ro, Sageun-dong, Seongdong-gu, Seoul, Republic of Korea

${ }^{s}$ Korea University, Department of Physics, 145 Anam-ro, Seongbuk-gu, Seoul 02841, Republic of Korea

${ }^{t}$ Kyung Hee University, 26 Kyungheedae-ro, Hoegi-dong, Dongdaemun-gu, Seoul, Republic of Korea

"Sungkyunkwan University,

2066 Seobu-ro, Jangan-gu, Suwon, Gyeonggi-do 16419, Seoul, Republic of Korea

${ }^{v}$ Benemerita Universidad Autonoma de Puebla, Puebla, Mexico

${ }^{w}$ Cinvestav,

Av. Instituto Politécnico Nacional No. 2508, Colonia San Pedro Zacatenco,

CP 07360, Ciudad de Mexico D.F., Mexico

${ }^{x}$ Universidad Iberoamericana, Mexico City, Mexico

${ }^{y}$ Sultan Qaboos University, Al Khoudh, Muscat 123, Oman

${ }^{z}$ National Centre for Physics, Quaid-i-Azam University, Islamabad, Pakistan

${ }^{a a}$ Department of Physics, Wisconsin University, Madison, WI 53706, United States

${ }^{b b}$ The British University in Egypt (BUE),

Elsherouk City, Suez Desert Road, Cairo 11837 - P.O. Box 43,Egypt

${ }^{c c}$ Department of Physics, Faculty of Science, Beni-Suef University, Beni-Suef, Egypt

${ }^{d d}$ School of Engineering, Damghan University, Damghan, 3671641167, Iran

ee INFN, Sezione di Pavia and University of Pavia, Via Bassi 6, IT-Pavia, Italy

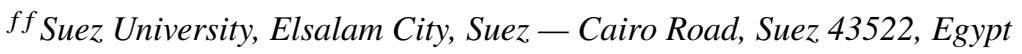

E-mail: Peicho.Petkov@cern.ch

Abstract: The CMS experiment has 1054 RPCs in its muon system. Monitoring their currents is the first essential step towards maintaining the stability of the CMS RPC detector performance. The current depends on several parameters such as applied voltage, luminosity, environmental conditions, etc. Knowing the influence of these parameters on the RPC current is essential for the correct interpretation of its instabilities as they can be caused either by changes in external conditions or by malfunctioning of the detector in the ideal case. We propose a Machine Learning(ML) based approach to be used for monitoring the CMS RPC currents. The approach is crucial for the 
development of an automated monitoring system capable of warning for possible hardware problems at a very early stage, which will contribute further to the stable operation of the CMS RPC detector.

KEYWORDS: Large detector-systems performance; Resistive-plate chambers 


\section{Contents}

1 Introduction 1

2 RPC current estimator 1

3 ML Model 2

4 Results 3

5 Conclusions 5

\section{Introduction}

The Compact Muon Solenoid (CMS) [1] experiment at the CERN Large Hadron Collider (LHC), uses Resistive Plate Chambers (RPC) [5] in the Muon system [3], together with Drift Tubes (DT) and Cathod Strip Chambers (CSC). RPC is the only muon detector in CMS present in both endcap and barrel regions (central and forward). RPCs operate in avalanche mode under non-uniform conditions such as high particle flux, radiation background, variations in atmospheric pressure, and other environmental parameters. There are 1056 chambers in the CMS RPC system (480 in the Barrel and 576 in the Endcaps) supplied by 781 high voltage (HV) channels. The stability of some of the most important detector performance parameters (such as efficiency and cluster size) is ensured by maintaining unvaried gas gain against environmental change, so-called PT-correction [6]. The PT-correction consists of adjusting HV values to compensate for variations in environmental pressure and temperature. The gas mixture composition and humidity are kept constant.

The stability of RPC currents is crucial for detector longevity. In this paper, we present a Machine Learning(ML)-based approach for current estimation. The aim of this method is to provide a reliable online monitoring system capable of spotting abnormal RPC current behavior. This system will help both shifters and experts, preventing operation in suboptimal conditions, which can cause detectors malfunctioning.

\section{RPC current estimator}

We assume that the current drawn by an RPC is uniquely determined by the design parameters, applied HV, gas mixture properties, environmental conditions and particles flux through the gas gaps. We propose the linearized RPC current estimator model assuming that variations in parameters are small:

$$
I_{\text {pred }}=C_{0}+C_{1} L_{\text {inst }}+C_{2} H V+C_{3} T+C_{4} L_{\text {inst }} e^{H V / P}+C_{5} R H+C_{6} P+C_{7} \Delta t,
$$

where the coefficients $C_{i}$ are to be determined by performing least squares fit to the historical data. $L_{\text {inst }}$ denotes the instantaneous luminosity of the LHC, $H V$ is the applied high voltage to the RPC 
gaps, $P$ is the atmospheric pressure, $T$ is the temperature, $R H$ is the relative humidity, and $\Delta t$ is the time interval since the beginning of the data taking in the given year.

The second term in eq. 2.1 accounts for the proportionality of the RPC current to the instantaneous luminosity, the third term is proportional to the ohmic current, the fourth and the seventh terms account for the variations in the residual temperature and pressure by the PT-correction, the sixth term corrects the current for variations in Bakelite resistivity due to changes of the environmental relative humidity, and the last term represents the tendency of the RPC current to increase in time with respect to the initial conditions for a given year.

\section{ML Model}

ML is widely used in a large variety of research fields. It encompasses a broad spectrum of algorithms and techniques devoted to extract conclusions from data. Therefore it is possible to construct models which can make predictions based on information extracted from the data. In the present paper, we use a regression model to predict the RPC current for a given set of conditions. According to eq. 2.1 the estimated RPC current $I_{\text {pred }}$ depends linearly on the model parameters $C_{i}$. Hence, we choose Generalized Linear Model (GLM) [8] with eight free parameters to predict the RPC current for given instantaneous luminosity, applied HV and environmental conditions. We used the Gaussian regression of the $\mathrm{H} 2 \mathrm{O} 3$ [9] GLM suite [10]. The flowchart of the model is represented in figure 1 . The input data to train and validate the model are taken from the CMS

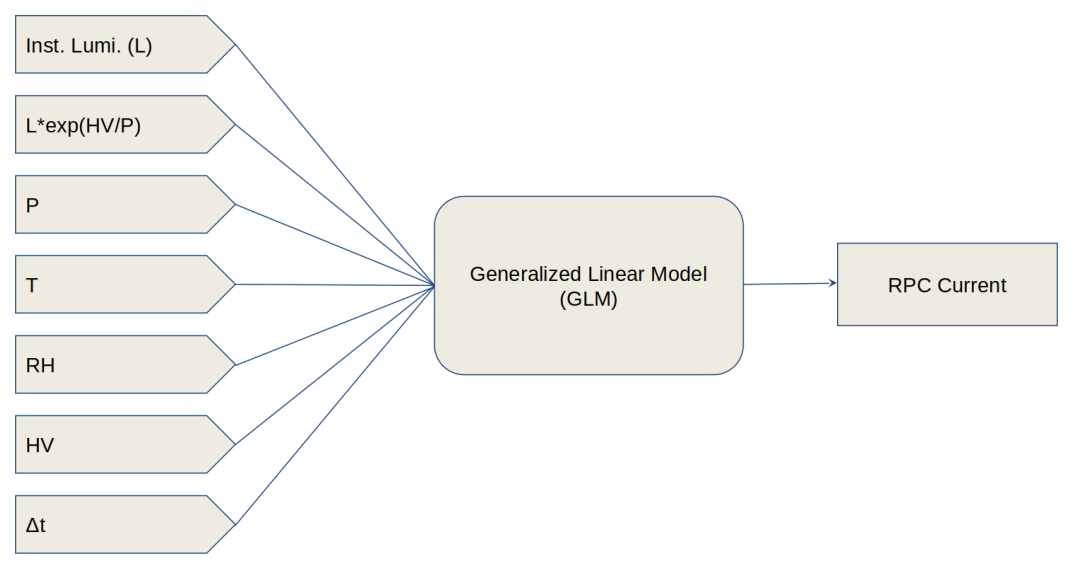

Figure 1. Flowchart of the ML-based RPC current prediction algorithm.

database. To extract information from the database we use a special tool developed by the CMS RPC collaboration called RPC current Automat [7]. It takes the data (RPC current, applied HV, and $\mathrm{UXC}^{1}$ environmental parameters) from the CMS Online Monitoring Database, synchronizes the data points with the RPC current timestamp and sets an additional flag encoding the LHC state and the HV supply module status. The LHC luminosity data [4] are added to those taken from RPC Current Automat forming the input dataset for training the GLMs.

The environmental pressure and relative humidity have a well defined seasonal behavior. Therefore, we selected a training and validation datasets in such a way that each dataset contained a

1The Compact Muon Solenoid detector complex is installed in the undergroud experimental cavern (UXC). 
period of at least one year of data-taking and the RPC chambers should have worked at least at two HV working points. Such conditions ensure proper models training to determine all parameters.

The HV is correlated with environmental parameters due to applied PT-correction and the fifth term in eq. 2.1 depends on the instantaneous luminosity, HV and pressure. GLM model is known to be robust to the overtraining problem because of the small number of parameters of the model [11].

\section{Results}

We tested the RPC current predicting models for 446 CMS Barrel RPC. The models were trained using 2017 data and validated on 2018 data. Both datasets satisfy the requirements defined in the previous section. The CMS Barrel RPCs consist of either two or three double gaps. Each chamber is supplied by a single HV channel. Therefore, the RPC currents discussed below are measured and analyzed per chamber. In figure 2, an example of RPC current prediction is shown which corresponds to the inner layer of the barrel muon station 3 in sector 3 of wheel +2 (namely W+2_S3_RB2in). It shows a good agreement between the prediction and the measured current value. The prediction follows the data points even though the HV working point was changed by about $200 \mathrm{~V}$.

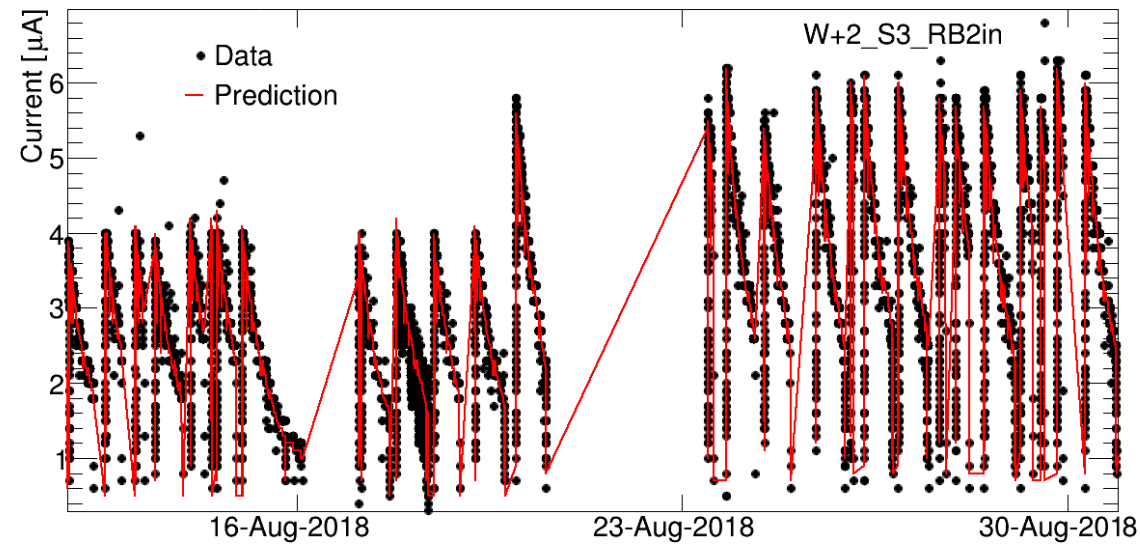

Figure 2. Predicted (red line) and measured (black points) current for RPC W+2_S3_RB2in although HV working point is changed by $200 \mathrm{~V}$ on 19 August 2018.

As it was already mentioned above, the RPC current is proportional to the instantaneous luminosity and the slope depends on the HV working point. As an example, figure 3a shows twodimensional histogram of the RPC current versus instantaneous luminosity for 2018 corresponding to an RPC in the negative $\phi$-part of barrel muon station 3 in sector 3 of wheel +2 (W+2_S3_RB3-). The corresponding GLM reproduces the current for the two HV working points correctly, as shown in figure 3b. The spread along the y-axis is due to variations in the other conditions. The RPCs operated at the lower HV working point during a very short period in 2018, about two weeks. This explains the smaller number of data points in the lower HV line, seen in the histogram on figure 3a. Moreover, during such a short period, the variations in the conditions stay relatively smaller compared to those of six months or longer periods of data taking, which results in different width of the corresponding HV lines in figure $3 b$. 


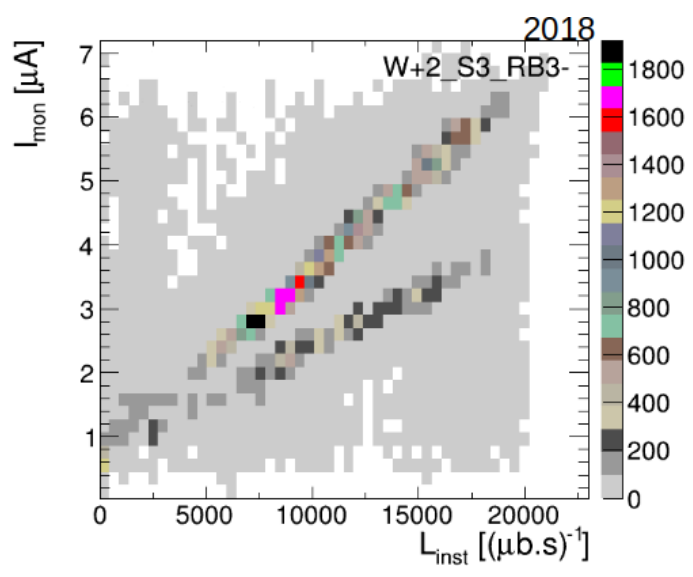

(a)

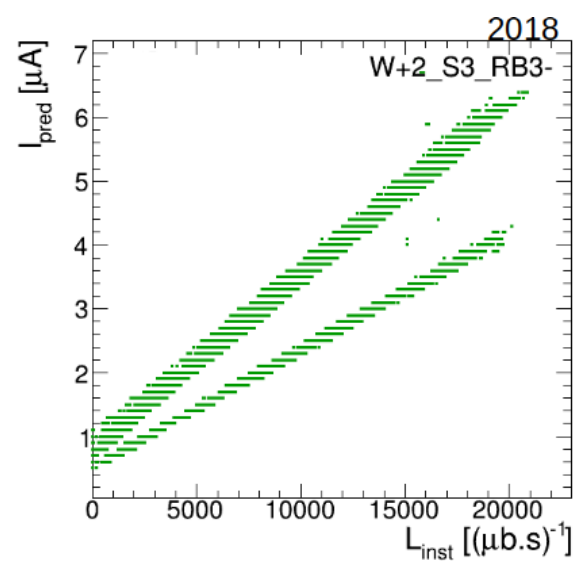

(b)

Figure 3. The monitored (a) and predicted (b) RPC current ( $\left.I_{\text {mon }}\right)$ vs. instantaneous luminosity $\left(L_{\text {inst }}\right)$ of RPC W+2_S3_RB3-. The datapoints are separated in two groups with different slopes due to chamber operation at two different $\mathrm{HV}$ working point in 2018.

The distribution of the average difference between measured and predicted RPC current for 2018 data is shown in figure 4. RPC current data were written to the database with an accuracy of $0.2 \mu \mathrm{A}$. According to that figure, we can conclude that the distribution is centered around 0 in the range of the RPC current data accuracy. The RMS of the distribution is $\approx 0.96 \mu \mathrm{A}$, which estimates the presented approach accuracy to be of the order of $1 \mu \mathrm{A}$. The RPCs in different muon stations operate in different background conditions [12]. The distributions of the average difference between monitored and predicted current for the RPCs in the four CMS Barrel Muon Stations (referred as RB1, RB2, RB3 and RB4) are presented in figure 5. The background in the innermost and the outermost stations RB1 and RB4, respectively is higher than in the rest of them. This leads to slightly wider distributions of average difference between monitored and predicted current in figure 5a and figure 5d compared to the distributions of the second and third Barrel Muon station shown in figure $5 \mathrm{~b}$ and figure $5 \mathrm{c}$, respectively.

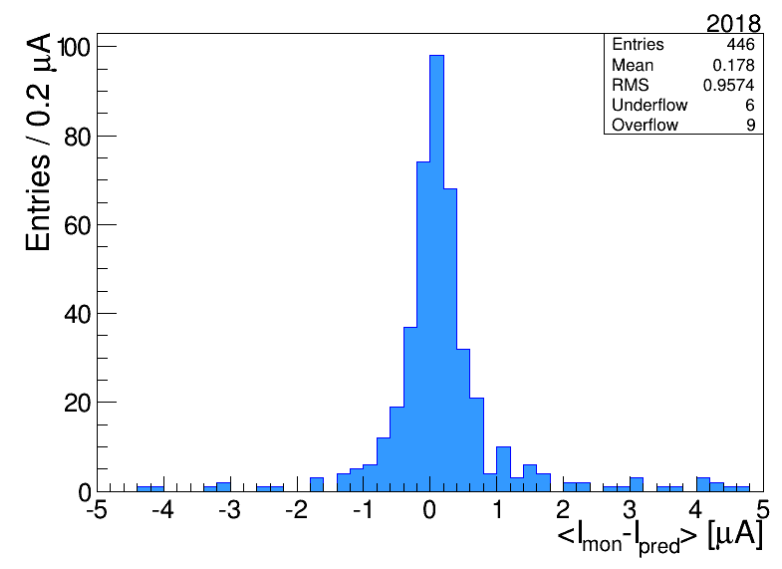

Figure 4. Distribution of the average difference between monitored and predicted current for 446 Barrel RPCs for 2018. 
The RPC chambers with an average difference between monitored and predicted current greater than $2 \mu \mathrm{A}$ are considered as problematic. Most of them are chambers with gas leaks. The rest are isolated cases of two kinds. First, with high average difference due to model training with inconsistent input data. This is a result of hardware changes. Second, the RPCs with stable but relatively high monitored current with respect to the predicted one.

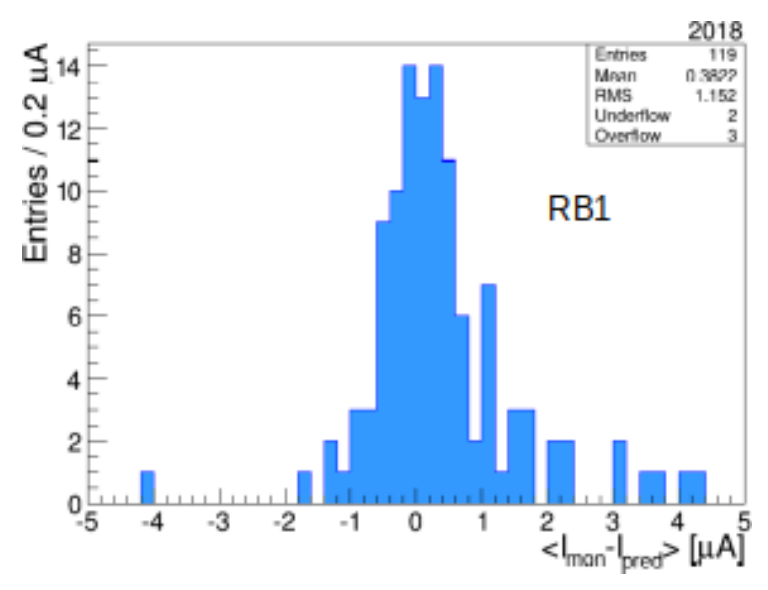

(a)

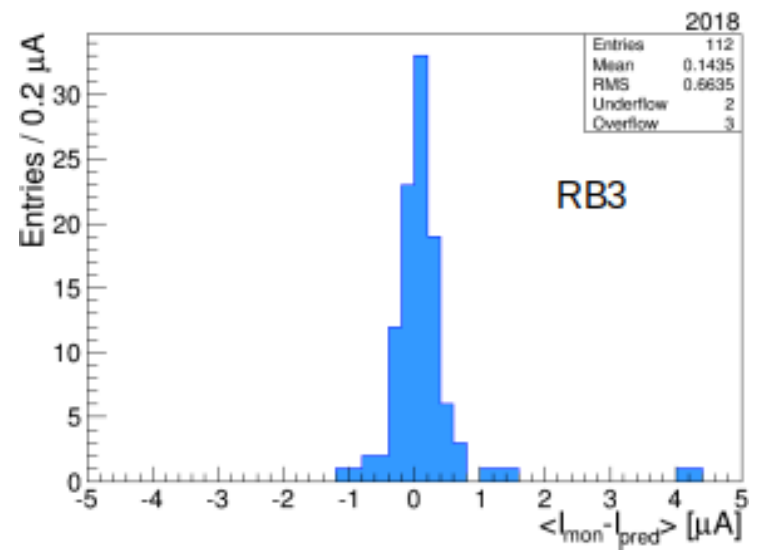

(c)

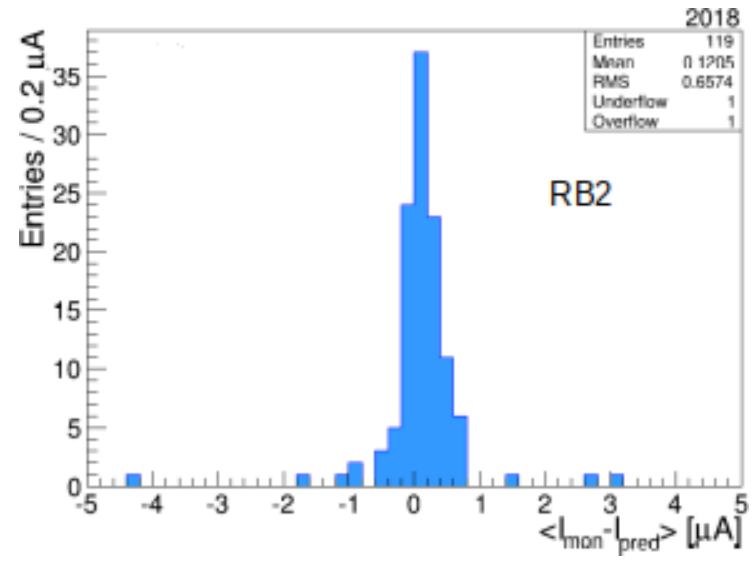

(b)

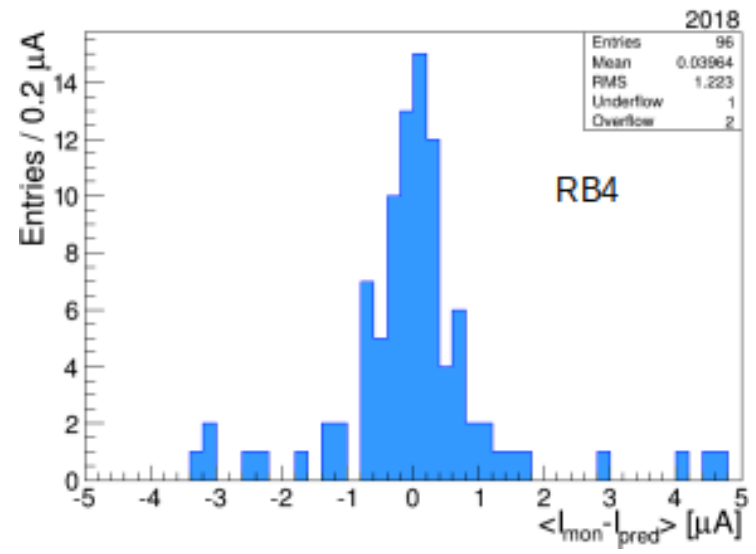

(d)

Figure 5. Distribution of the average difference between monitored and predicted current for CMS Barrel RPC in muon stations: RB1 (a), RB2 (b), RB3 (c) and RB4 (d).

\section{Conclusions}

We developed a ML-based approach for predicting RPC currents. It was tested on $446 \mathrm{CMS}$ Barrel RPC and the validation showed overall accuracy of about $1 \mu \mathrm{A}$. The model was able to find RPC chambers with known hardware problems and some RPC chambers with unusual current trends to be carefully monitored. The tool is used for historical analysis of the CMS RPC currents and can be used for predicting RPC currents behavior in the future runs. The prediction power of the model is expected to be improved by including more input variables. 


\section{Acknowledgments}

This paper is partially supported by the Bulgarian Ministry of Education and Science (contract D01-205/23.11.2018) under the National Science Program "Information and Communication Technologies for a Single Digital Market in Science, Education and Security (ICTinSES)", approved by DCM\#577/17.08.2018. We would like to thank especially to Anton Dimitrov and Osvaldo Miguel Colin who mainly developed the RPC Condition Data Automation concept and implementation respectively, and all our colleagues from the CMS RPC group and L1 muon trigger group for their dedicated work to keep the stable performance of the RPC system. We wish to congratulate our colleagues in the CERN accelerator Department for the excellent performance of the LHC machine. We thank the technical and administrative staff at CERN and all CMS institutes.

\section{References}

[1] CMS collaboration, The CMS Experiment at the CERN LHC, 2008 JINST 3 S08004.

[2] CMS collaboration, Performance of the CMS muon detector and muon reconstruction with proton-proton collisions at $\sqrt{s}=13 \mathrm{TeV}, 2018$ JINST 13 P06015 [arXiv: 1804.04528].

[3] CMS collaboration, The CMS muon project: Technical Design Report, Technical Design Report CMS, CERN-LHCC-97-032, CERN, Geneva (1997).

[4] CMS collaboration, CMS luminosity measurement for the 2017 data-taking period at $\sqrt{s}=13 \mathrm{TeV}$, Tech. Rep., CMS-PAS-LUM-17-004, CERN, Geneva (2018).

[5] M.A. Shah et'al., The CMS RPC Detector Performance and Stability during LHC RUN-2, 2019 JINST 14 C11012 [arXiv: 1808. 10488].

[6] S. Colafranceschi et al., Performance of the Gas Gain Monitoring system of the CMS RPC muon detector and effective working point fine tuning, 2012 JINST 7 P12004.

[7] O.M. Colin, CMS RPC Condition Data Automation, talk given at the XIV Workshop on Resistive Plate Chambers and Related Detectors (RPC 2018), https://indico.cern.ch/event/644205/contributions/2862294/.

[8] P. McCullagh and J.A. Nelder, Generalized Linear Models., Chapman \& Hall/CRC., London (1989)

[9] https://docs.h2o.ai/h2o/latest-stable/h2o-docs/welcome.html.

[10] https://docs.h2o.ai/h2o/latest-stable/h2o-docs/data-science/glm.html.

[11] K.A. McQuisten and A.S. Peek, Comparing artificial neural networks, general linear models and support vector machines in building predictive models for small interfering RNAs, PLoS ONE 4 (2009) e7522.

[12] R. Hadjiiska et al., CMS RPC Background - Studies and Measurements, in 15th Workshop on Resistive Plate Chambers and Related Detectors, 5, 2020 [arXiv: 2005 . 12769]. 